\title{
Fabrication, morphologies and properties of luminescent PMMA porous nano-fibers
}

\author{
Haiying Xia, Fulai Zhao, Li Gao, Chaohua Wang, Peng Xi* \\ Tianjin Municipal Key Laboratory of Fiber Modification and Functional Fiber, Tianjin Polytechnic \\ University, 399 Bin Shui West Road, 300387 Tianjin, PR China \\ xiahaiying2016@163.com ialufhz@163.com 13752222781@163.com 2286101696@qq.com \\ xpsyq0007@sina.com
}

\begin{abstract}
KEYWORD: Porous; luminescent; PMMA; Ultrahigh surface area
ABSTRACT: Luminescent poly(methyl methacrylate) (PMMA) porous fibers were prepared by electrospinni-ng technology using a binary solvent system of EA/DMF and DCM/DMF. The effects of the ambient humidity, content of rare earth complex ( $\mathrm{Tb}(\mathrm{phen}) \mathrm{L} 3)$, molecular weight of PMMA, and the proportion of E-A/DMF (mass ratio) on the pore formation of the fibers were investigated. The morphology, structure, surface area, and luminescent properties of porous luminescent fibers were characterized. Results showed that when using EA/DMF as mixed solvent system, the solvent ratio has great influence on the pore formation of fibers comparing to the effect of environmental humidity. PMMA fibers with higher molecular weight only have pores on the surface, while relative low molecular weight's fibers have pores both inside and on the surface. The addition of Tb(phen)L3 can improve the morphology of fibers, but the excess will hinder the formation of pores. Due to the effect of porous formation, the surface area of fiber mat increased by $113 \mathrm{~m} 2 / \mathrm{g}$. The as-prepared fibers have good luminescent properties, and high specific surface area. The change in fluorescence intensity affected by water and oil has potential application in oil water separation indicator.
\end{abstract}

\section{INTRODUCTION}

Electrospinning technology, as a simple, controll-able and versatile technique for generating fibers with diameters on both the micro and nano scales, has recently made significant progress in producing uniform fibers with novel compositions and morpho-logies. For the virtue of a high surface area in mass ratio, nonwoven electrospun fiber mat has afforded potentially advantageous performance in the fields encompassing bioengineering, tissue engineering to membrane science, electrochemistry, and environm-ental engineering[1,2]. Meanwhile electrospun poro-us fibers can be applied in chemical filtration, drug molecules controlled release, oil/water separation, solid support catalysts, high performance sensors, scaffolds for tissue/cell growth, carriers for immobi-lizing biological and pharmacologically significant agents and molecules $[3,4,5]$.

As for the development of porous morphology, the formation of porous fiber was first reported by Casper et al. [6] who demonstrated that surface features or pores became evident when electrospinning of PS/THF in an atmosphere with more than 30\% relative humidity. Increasing humidity causes an increase in the number, diameter, shape, and distribution of the pores. Electrospinning of the polymer solution in the humid environment (i.e., 60\% humidity) can produce the porous structure. Increasing the molecular weight of the polystyrene (PS) results in larger, less uniform shaped pores. In the present case, the porous fiber can be spun by a evaporable solvent such as MC or THF. It is well documented that the solvent quality can be easily reduced by the surrounding moisture, which in turn, could drive liquid-liquid-phase separation leading to the porous fiber formation[7]. One possible account is that both MC and THF are known to be water solu-

ble and thus would be sensitive to a minute level of moisture. The reduction in solvent quality due to such moisture absorption of the solvent will open up the upper critical solution temperature (UCST) gap which eventually would trigger the phase separation during electrospinning. Porous surface morphologies [6,8-11] have been observed in electrospun fibers from solution in a evaporable solvent; this surface porosity can be varied by controlling the relative humidity of the environment and the molecular weight of the polymer. The simulations of Kyu and co-workers[1,12-13] predicted morphologies that ranged from smooth hollow fibers, to fibers with a smooth surface and porous core, to fibers with porous morphologies both at the fiber surface and in the core. Chia-Ling Pai et al. [14] experimentally confirmed the electrospun fibers exhibiting a smooth fiber surface and porous 
interior. They found that fibers with a smooth surface and porous. Interiors, rather than a homogeneous consolidated solid struc-ture, are readily obtained when the system is electro-spun in a very humid environment.

Although researchers have prepared some porous PMMA fibers, the fabrication of high surface area luminescent porous PMMA fibers has not been reported. In this study, we fabricated a new kind of PMMA porous fibers with luminescent properties by electrospinning, using a binary solvent system of EA/DMF. The $\mathrm{Tb}$ (phen) $\mathrm{L}_{3}$ (phen=1,10-phenanthroli-ne, $\mathrm{L}=\mathrm{p}$-methoxy benzoic acid) complexes was intr-oduced and endow as-prepared fiber with excellent fluorescent properties. We discussed the effects on the porosity of as-prepared porous fibers, such as environmental humidity, molecular weight values of PMMA, the rate of EA/DMF and the concentration of $\mathrm{Tb}$ (phen) $\mathrm{L}_{3}$. Basing on these effects, we prepared the luminescent porous PMMA fibers with brilliant porosity, ultrahigh surface area and great fluorescent properties.

\section{EXPERIMENTAL}

\section{Materials}

Commercial PMMA, $(\mathrm{Mw}=590000$ and $\mathrm{Mw}=$

110000) were purchased from Mitsubishi Engineeri-ng-Plastic Corporation. Europium(III) oxide (99.95\%, AR) was bought from Shanghai Yuelong Nonferrous Pioneer Metals Corporation. pmethoxy benzoic acid was from China National Medicines Corporation Ltd. and 1,10-phenanthroline (phen, AR

) from Beijing Chemical Works. N,N-dimethylform-amide (DMF, AR) and ethyl acetate (EA, AR) were purchased from Tianjin Kemiou Chemical Reagent Co. Ltd. and Tianjin Fengchuan Chemical Reagent Technology Co. Ltd. , respectively. All chemicals were directly used as received without further purification.

\section{Preparation of $T b(p h e n) L_{3}$ complexes}

P-methoxybenzoic acid (L), 1,10-phenanthroline (Phen) and $\mathrm{Tb}\left(\mathrm{NO}_{3}\right)_{3}$ were dissolved in ethanol to the chemical formula $\mathrm{Tb}($ phen $) \mathrm{L}_{3}$ molar ratio, respectiv-ely and forming ligand solution of $0.1 \%$. In $50 \sim 60^{\circ} \mathrm{Cwater}$ bath, the solution was stirred for $30 \mathrm{~min}$. And then, the solution was adjusted $\mathrm{pH}$ to 8.5 using $\mathrm{NH}_{3} \cdot \mathrm{H}_{2} \mathrm{O}$. After $24 \mathrm{~h}$, a white precipitate was achiev-

ed. The precipitate was washed with ethanol and water for 3 times, respectively. Finally drying $12 \mathrm{~h}$ in vacuum at $80{ }^{\circ} \mathrm{C}$, the terbium complexes $\left(\mathrm{Tb}(\mathrm{phen})-\mathrm{L}_{3}\right)$ was obtained[17].

\section{Preparation of PMMA/Tb(phen) $\mathrm{L}_{3}$ composite porous fibers}

$\mathrm{Tb}$ (phen) $\mathrm{L}_{3} / \mathrm{PMMA} / \mathrm{DMF} / \mathrm{EA}$ polymer solution was prepared by adding measured $\mathrm{Tb}$ (phen) $\mathrm{L}_{3}$ and PMMA into different proportional EA/DMF solvent, and stirring $2 \mathrm{~h}$ at $70{ }^{\circ} \mathrm{C}$. Then the solution was elec-

trospun by self-made electrostatic spinning device composed of injection pump, high-voltage power supply and collector. Finally the corresponding $\mathrm{Tb}(\mathrm{p}-\mathrm{hen}) \mathrm{L}_{3} / \mathrm{PMMA}$ luminescent fibers was obtained. Te-

mperature and humidity were $20{ }^{\circ} \mathrm{C}, 40 \%-60 \%$, respectively.

\section{Characterization methods}

The composition and structure, morphology, and fluorescent properties of the samples were character-ized by field emission scanning electron microscopy (FE-SEM) (Hitachi S4800, Japan), Surface Area \& Pore Size Analyzer(CFP-1100-A, USA), fluorescen-ce spectrometer ( Gangdong F3800 ) and ultraviolet analyzer(Yuhua ZF7). 


\section{RESULTS AND DISCUSSION}

\section{Effects of the rate of EA/DMF on the porosity of $\mathrm{Tb}$ (phen) $\mathrm{L}_{3} / \mathrm{PMMA}$ fiber}
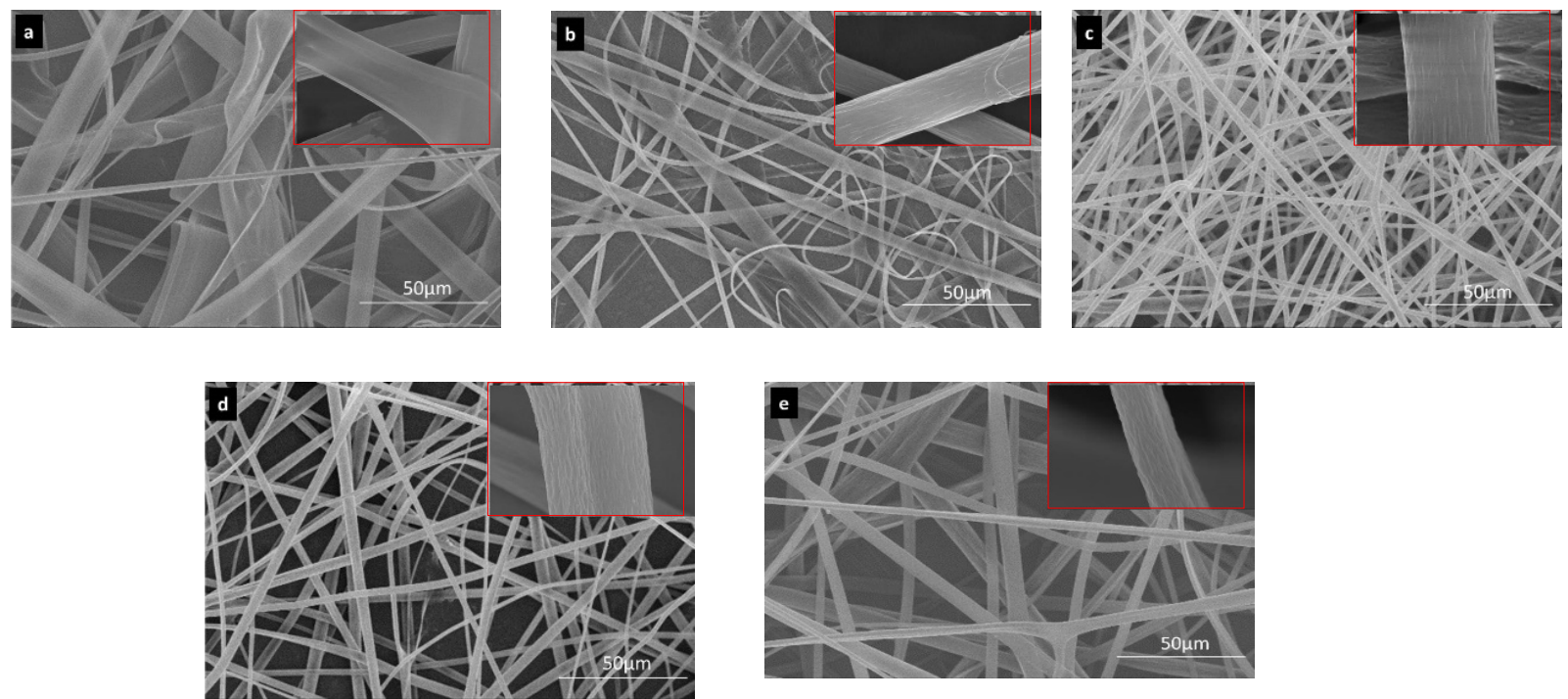

Figure 1. FE-SEM images of the electrospun

$\mathrm{Tb}$ (phen) $\mathrm{L}_{3} / \mathrm{PMMA}$ fibers obtained under different solvents ratios. a 1:1, b 3:1, c 5:1, d 7:1, e 9:1.

Figure 1 shows the morphologies of as-prepared $\mathrm{Tb}(\mathrm{phen}) \mathrm{L}_{3} / \mathrm{PMMA}$ fiber under different rate of EA/DMF. As it can be seen from the images, the number of pores will gradually increase with the addition of EA in the mixture solvent. Phase separation based on the evaporation of solvents is the main mechanism behind the formation of porous fibers $[18,19]$. The evaporation rate of EA is faster than DMF. When a more volatile solvent is used in the electrospinning process, solvent-rich regions begin to form. The solvent-rich regions are transfor-med into pores as soon as the solvents are evaporat-ed. DMF is less volatile, so the number of pores grows with the increase of EA in the mixture solvent.

\section{Effects of different molecular weight of PMMA on the porosity of $\mathrm{Tb}(\mathrm{phen}) \mathrm{L}_{3} / \mathrm{PMMA}$ fiber}
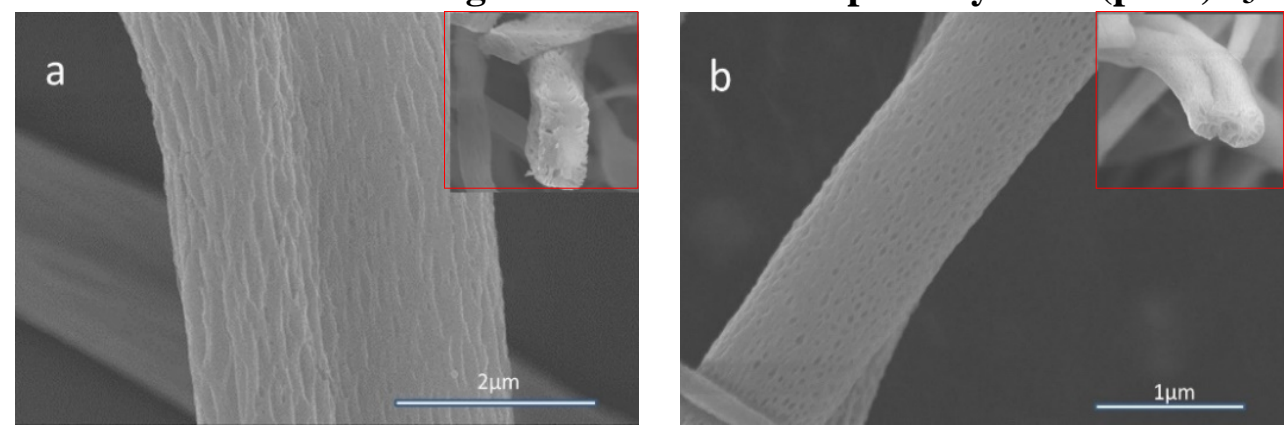

Figure 2. FE-SEM images of the electrospun $\mathrm{Tb}(\mathrm{phen}) \mathrm{L}_{3} / \mathrm{PMMA}$ porous fibers with different molecular weight values. $\mathrm{a}: \mathrm{Mw}=590000 ; \mathrm{b}: \mathrm{Mw}=$

110000.

It is evident in figure 2 that under the same spinning parameters, the pores on the fibers with low molecular weight $(\mathrm{Mw}=110000)$ are small and uniform. Look at the cross-sectional of porous fibers, there are also many pores inside of the fibers. As to the pores on the fibers with high molecular weight $(\mathrm{Mw}=590000)$, they are large, non-circular and non-uniform, and there is no pores inside of the fiber. As the molecular weight of the polymer increases, both the diameter of the pores and the distribution of the pore are increase. Theses possible reasons is that, the rapid evaporation of solvent decreases the temperat-ure of jets in the high speed moving of liquid jets, so as to cause thermally induced phase separation. The result of phase separation is to form a polymer enrichment region and a solvent rich areas. The change of molecular weight affects the competition between phase separation and curing of the liquid jets under the influence of complicated external electric field. As for the in- 
fluence of polymer molecular weight on the morphology of the fibers in electrospinning process, the research of predecessors showed that the viscosity of polymers reduced with the decrease of molecular weight, because of the relatively short molecular chains and low degree of entanglement of polymers. In the spinning process, the solvent molecules of polymer solution with low molecular weight can be easily removed [20]. For low molecular weight polymer solution, the speed of phase separation on the surface of the liquid jets is higher. And thanks to the drastic movement, the elongation forces on the jet are larger. There's not enough time to form a complete entity. Rapid phase separation, curing and elongation forces make the fiber surface and internal becoming pores due to solvent evaporation. On the contrary, high molecular weight polymer solution has longer chains, higher chain entanglement degrees and viscosity. The speed of phase separation of the liquid jets is lower. It is easier to form a complete entity but not porous channel.

\section{Effects of the Concentration of $\mathrm{Tb}($ phen $) \mathrm{L}_{3}$ on the porosity of $\mathrm{Tb}(\mathrm{phen}) \mathrm{L}_{3} / \mathrm{PMMA}$ fiber}
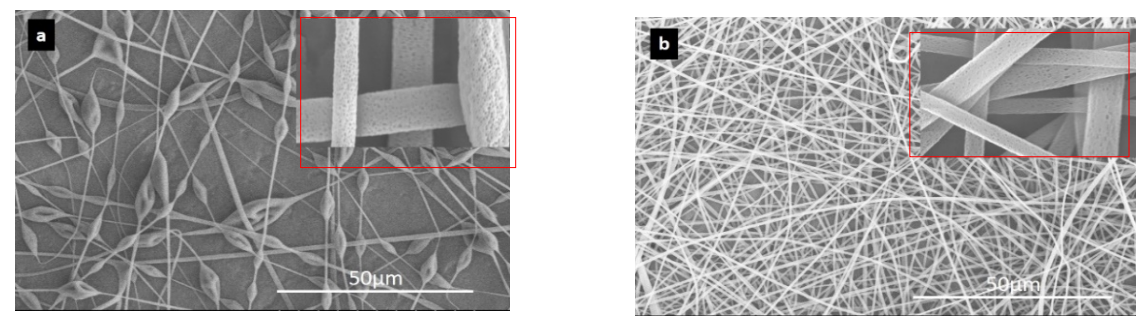

Figure 3. FE-SEM images of porous fibers with different $\mathrm{Tb}$ (phen)L3 content. a : $0 ; \mathrm{b}: 1 \%$;

Figure $3 \mathrm{a}$ and $\mathrm{b}$ are the eletrospun fibers of PMMA with 14\% solid content and the molecular weight is 110000 . The spinning parameters of them are unanimous. The difference between them are that $1 \%$ wt of $\mathrm{Tb}$ (phen) $\mathrm{L}_{3}$ was added to $\mathrm{b}$ sample and a sample was not. Obviously that the introduction of $\mathrm{Tb}$ (phen) $\mathrm{L}_{3}$ can decrease the beadlike structure , and improve the spinnability of porous fibers.

In the electrospinning process, the addition of $\mathrm{Tb}($ phen $) \mathrm{L}_{3}$ incurred a higher charge density on the surface of the solution jet, bringing more electric charges to the jet. As the charges of the jet increased, higher elongation forces were imposed to the jet under the electrical field, resulting in smaller bead and thinner fiber diameters [21]. However, it can be seen from Figure 4 that the pores on the fibers are gradually decreased with the increase of $\mathrm{Tb}(\mathrm{phen}) \mathrm{L}_{3}$ content.
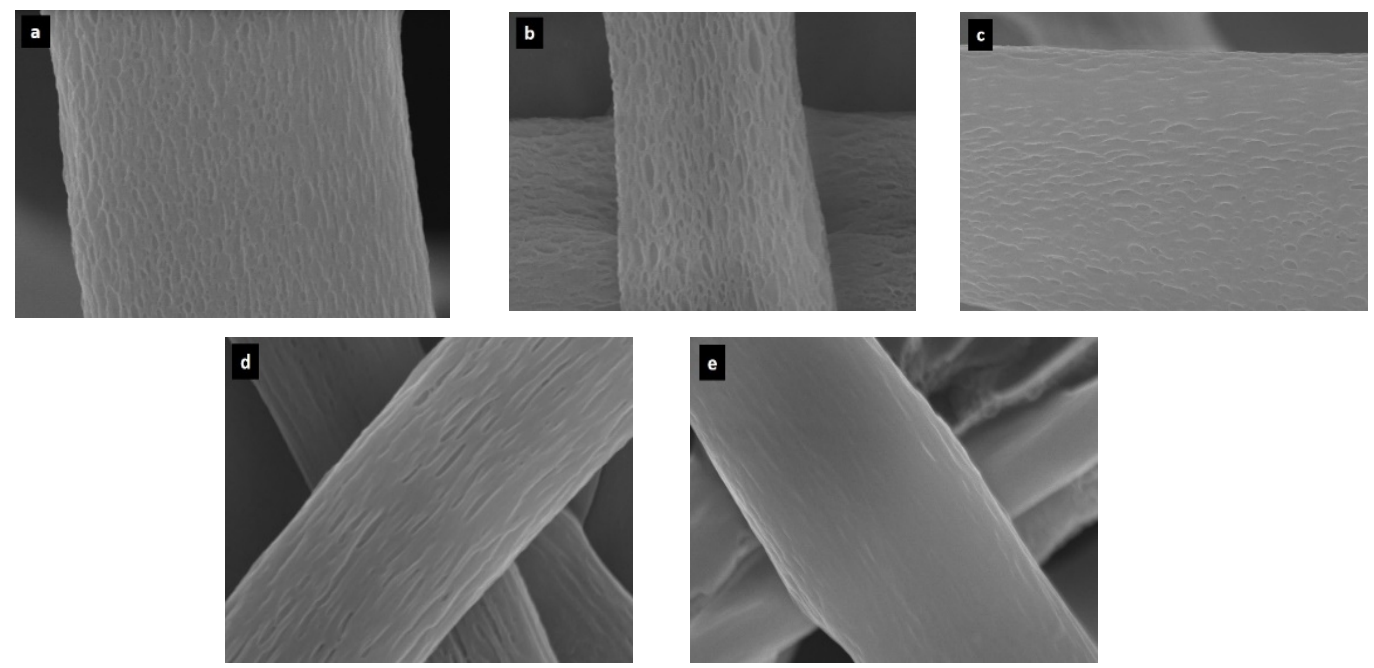

Figure 4. FE-SEM images of porous fibers with different $\mathrm{Tb}(\mathrm{phen}) \mathrm{L}_{3}$ content. a-e: $0.5 \%, 1 \%$, $1.5 \%, 2 \%, 2.5 \%$. 


\section{Analysis of BET surface area}
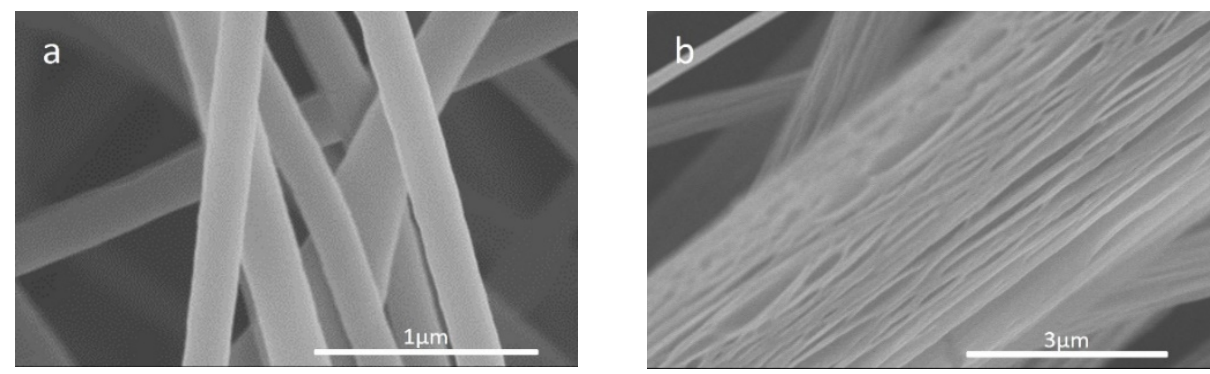

Figure 5. FE-SEM images of $\mathrm{Tb}$ (phen) $\mathrm{L}_{3}$ /PMMA fibers with different morphology. a: nonporous; b: porous.

As shown in the figure $5, \mathrm{a}$ and $\mathrm{b}$ are the images of nonporous and porous fibers. The surface area of them are $300.96 \mathrm{~m}^{2} / \mathrm{g}$ and $414.49 \mathrm{~m}^{2} / \mathrm{g}$, respectively. The increase in the surface area of the porous fiber membrane may be due to the formation of pores.

\section{Fluorescence Spectra Analysis}

In order to research the effect of pores on the photoluminescent (PL) properties of $\mathrm{PMMA} / \mathrm{Tb}$ (phen) $\mathrm{L}_{3}$ fibers, nonporous and porous $\mathrm{PMMA} / \mathrm{Tb}\left(\right.$ phe-n) $\mathrm{L}_{3}$ fibers were analyzed through fluorescence spectrum test.
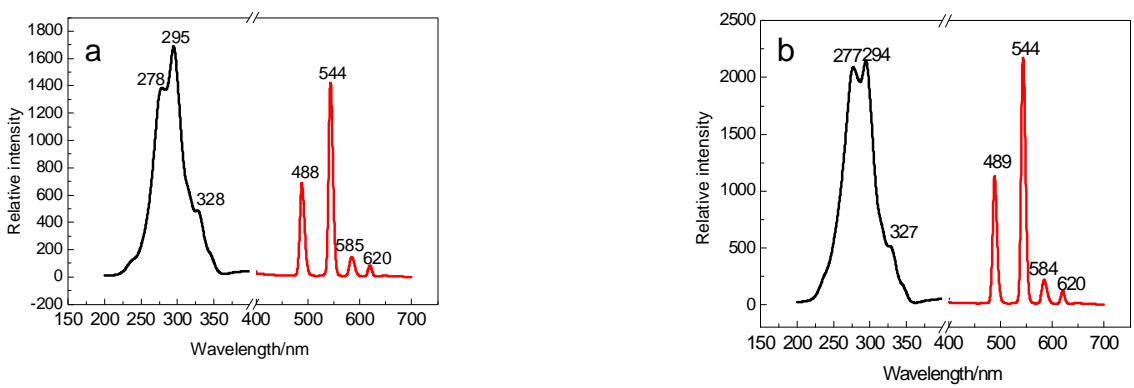

Figure 6. Fluorescence spectra of nonporous

and porous fibers. a: nonporous; $b$ : porous.

Figure 6 showed fluorescence spectra of $\mathrm{PMM}-\mathrm{A} / \mathrm{Tb}$ (phen) $\mathrm{L}_{3}$ composite fibers. As for $\mathrm{a}$ and $\mathrm{b}$, the left side graphs were the excitation spectra of the samples. When the monitoring wavelength was 544 $\mathrm{nm}$, the broad excitation bands extending from 200 to $360 \mathrm{~nm}$ were observed. The right side graphs in the Figure 6 were the emission spectra of the samples. It was found that under the excitation of $295 \mathrm{~nm}$ ultraviolet light, the sample exhibited predo-minant peaks at 489,544,584 and $620 \mathrm{~nm}$ attributed to the ${ }^{5} \mathrm{D}_{4} \rightarrow{ }^{7} \mathrm{~F}_{6},{ }^{5} \mathrm{D}_{4} \rightarrow{ }^{7} \mathrm{~F}_{5},{ }^{5} \mathrm{D}_{4} \rightarrow{ }^{7} \mathrm{~F}_{4}$ and ${ }^{5} \mathrm{D}_{4} \rightarrow{ }^{7} \mathrm{~F}_{3}$ transitions, especially $544 \mathrm{~nm}$ emission peak is the strongest. At $544 \mathrm{~nm}$, the peak values of nonporous and porous fibers are 1412 and 2160, respectively.

Due to the coarsening fibers, rare earth complex-es distribute much non-uniformly. And the porous morphology of fiber surface makes the light easily diffuse reflection, thus the measured fluorescence intensity becomes high. That is why the fluorescence intensity of nonporous fibers is lower than that of porous. Even so, the fluorescent property of porous fibers is excellent.

\section{Potential application in oil water separation indicator}

To investigate the influence of oil and water on the luminescent of $\mathrm{PMMA} / \mathrm{Tb}$ (phen) $\mathrm{L}_{3}$ composite porou-s fibers, the fiber mat was cut into several slices. One slice was chosen as sample "a". A cup of $80 \mathrm{~g}$ water was filled in advance. Before sample "a" was thrown into the water, $0.01 \mathrm{~g}$ engine oil was added into the water. Then keep stirring to make the slice in oil/water for $30 \mathrm{~s}$. Afterwards get the sample "a" out of water. For sample "b", still, was thrown into a cup of water with a second $0.01 \mathrm{~g}$ engine oil and stirred for $30 \mathrm{~s}$. Then, Sample "b" was gained. In the similar fashion, sample "c" and "d" were obtained. The fluorescence intensity of samples was shown in Figure 7. By comparison, another series of samples were obtained by throwing slice into the water without oil. The steps were the same as described above. The fluorescence intensity of these samples was shown in Figure 8. 

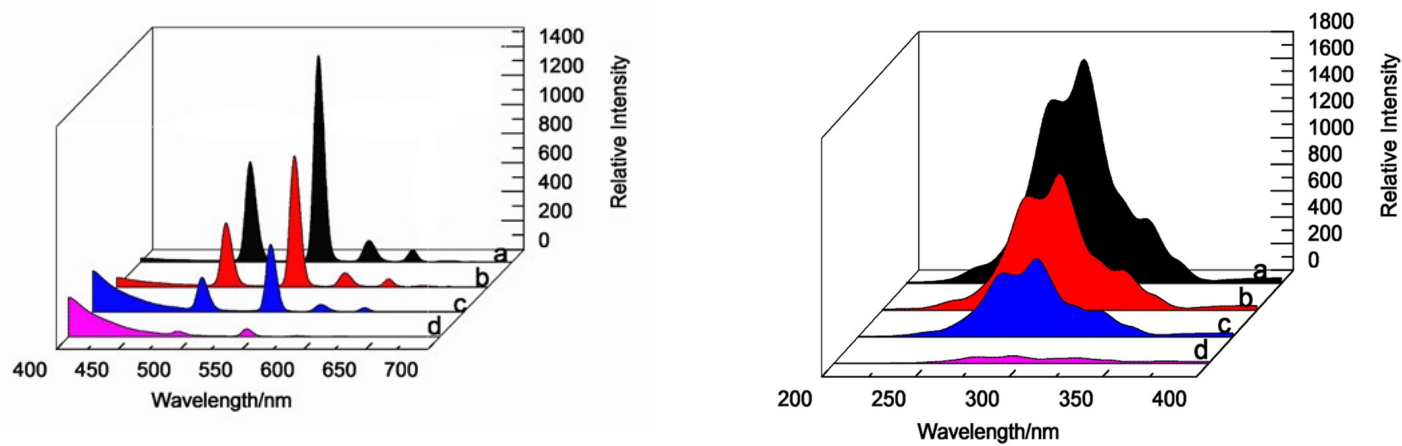

Figure 7. Fluorescence spectra images of different oil absorption.
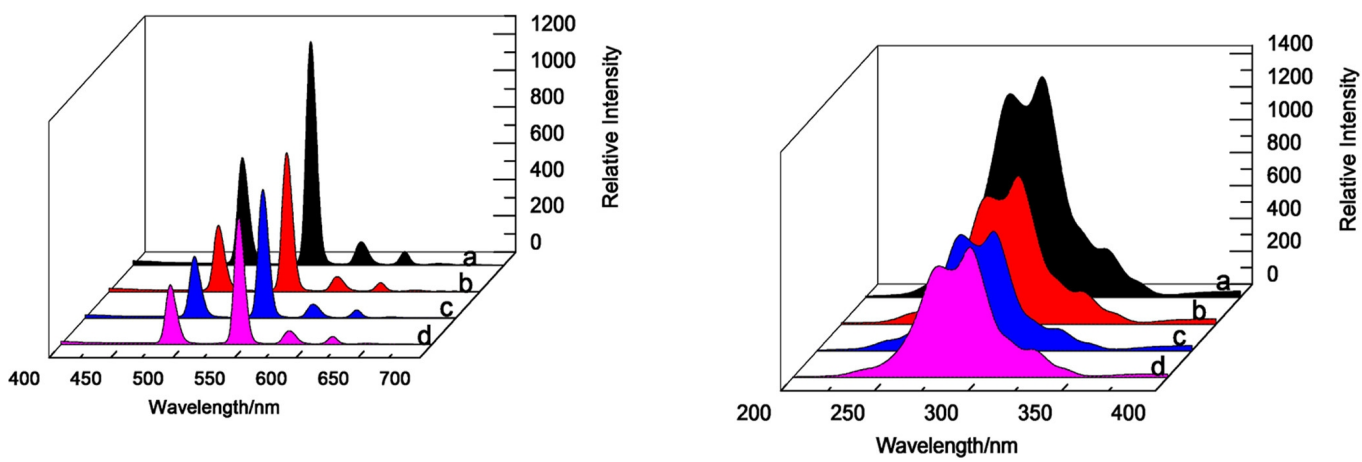

Figure 8. Fluorescence spectra images of different water absorption.

Comparing Figure 7 with Figure 8, we can concl-ude that the fluorescence intensity of PMMA porous fiber mat is more easily affected by oil than water. With the absorption of oil, the fluorescence intensity decrease rapidly, even near to zero. That is mainly because PMMA has the lipophilic and hydrophobic structure, oil is easier to be absorbed.

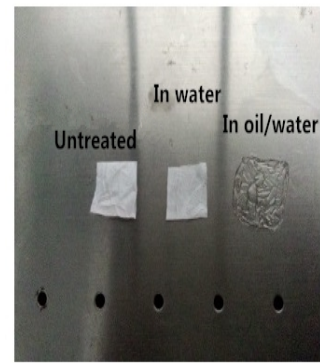

Under natural light

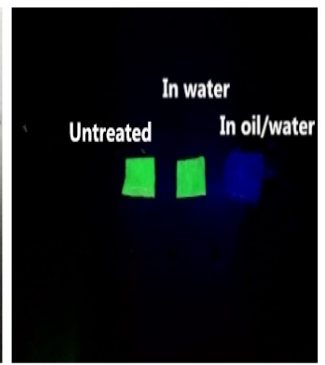

Under ultraviolet light

Figure 9. Picture of samples under natural and ultraviolet light.

As is shown in Figure 9, three pieces of porous $\mathrm{PMMA} / \mathrm{Tb}$ (phen) $\mathrm{L}_{3}$ fiber mats-untreated, 5 minutes in water, and 5 minutes in oil/water, were irradiated by natural and ultraviolet light, respectively. Under natural light, mat treated in water for 5 minutes has little change, while the mat in oil/water for 5 minutes became almost transparent. On the other hand, under ultraviolet light, mat treated in water for 5 minutes emitted green light nearly the same to the untreated, while in oil/water emitted the color of ultraviolet light. That is, the fluorescence of mat treated in oil/water disappeared. This special property can be used to indicate the effect of oil water separation.

\section{CONCLUSION}

In conclusion, nonporous and porous electrospun $\mathrm{PMMA} / \mathrm{Tb}(\mathrm{phen}) \mathrm{L}_{3}$ fiber membranes were prepared by controlling the ratio of EA/DMF during the electrospinning of a PMMA polymer solution. The solvent ratio has great influence on pore of the fibers comparing to the effect of environmental humidity. PMMA fibers with higher molecular weight have only pores on the surface, while fibers 
with lower molecular weight have pores both inside and on the surface. The addition of $\mathrm{Tb}(\mathrm{phen}) \mathrm{L}_{3}$ can improve the morphology of fibers, the excess will hinder the formation of pores. The fibers we finally prepared have good luminescent properties, and high specific surface area. The change of fluorescence intensity affected by water and oil has potential application in oil water separation indicator.

\section{Acknowledgement}

This research was financially supported by the National Natural Science Funds (51203112), National Natural Science Funds (51373118), Application Fundamental and Advanced Technology Research Proposal Project of Tianjin, China (13JCYBJC17200).

\section{References}

[1] P. Dayal, J. Liu, and S. Kumar. Macromolec-ules, 2007, 40 (21): 7689-7694.

[2] L. Zhou, Q. Cao, and B. Jing. Journal of Power Sources, 2014, 263: 118-124.

[3] X. S. Li, and G. Y. Nie. Chinese Science Bulle-tin, 2004, 49 (21): 2368-2371.

[4] L. F. Zhang, and Y. L. Hsieh. Nanotechnology, 2006, 17 (17): 4416.

[5] S. C. Moon, and J. K. Choi. Fibers and Polym-ers, 2008, 9 (3) : 276-280.

[6] C. L. Casper, and J. S. Stephens. Macromolec-ules, 2004, 37 (2): 573-578.

[7] H. Matsuyama, M. Teramoto, and R. Nakatani. Journal of applied polymer science, 1999, 74 (1): 159-170.

[8] S. Megelski, and J. S. Stephens. Macromolecules, 2002, 35 (22): 8456-8466.

[9] M. Bognitzki,W. Czado, and T. Frese. Advanc-ed Materials, 2001, 13 (1): 70-72.

[10] J. P. Jeun, Y. H. Kim, and Y. M. Lim. Journal of Industrial and Engineering Chemistry, 2007, 13 (4): 592-596.

[11] Y. Miyauchi, B. Ding, and S. Shiratori. Nanote-chnology, 2006, 17 (20): 5151.

[12] P. Dayal, T. Kyu. Journal of applied physics, 2006, 100 (4): 043512.

[13] A. J. Guenthner, S. Khombhongse, and W. Liu. Macromolecular theory and simulations, 2006, 15 (1): 87-93.

[14] C. C. Pai , M. C. Boyce. Macromolecules,2009, 42 (6): 2102-2114.

[15] C. C. Kuo, and C. H. Lin. Macromolecules, 2007, 40 (19): 6959-6966.

[16] H. S. Bae, and A. Haider. Journal of Polymer Research, 2013, 20 (7): 1-7.

[17] Z. H. Gao, R. F. Wang, and S. K. Shi. Chinese Journal of Luminescence, 2005, 26 (3): 344-348 (in Chinese).

[18] T. Subbiah, G. S. Bhat, and R. W. Tock. Journal of Applied Polymer Science, 2005, 96 (2): 557-569.

[19] S. Ramakrishna, K. Fujihara, and W. E. Teo. Mat Today, 2006, 9 (3): 40-50.

[20] A. Ziabicki. Wiley, New York, 1976: 159-251.

[21] Z. M. Huang, and Y. Z. Zhang. Composites science and technology, 2003, 63 (15): 2223-2253. 